\title{
EVALUATIVE INQUIRY: ENGAGING RESEARCH EVALUATION ANALYTICALLY AND STRATEGICALLY
}

\author{
SARAH DE RIJCKE, TJITSKE HOLTROP, WOLFGANG KALTENBRUNNER, JOCHEM ZUIJDERWIJK, ANNE BEAULIEU, \\ THOMAS FRANSSEN, THED VAN LEEUWEN, PHILIPPE MONGEON, CLIFFORD TATUM, GOVERT VALKENBURG AND \\ PAUL WOUTERS
}

DOI: 10.22163/fteval.2019.386

\section{ABSTRACT}

$\mathrm{T}$ raditional frameworks for academic evaluation are focused on registering the achievements of research units' academic and societal achievements. These frameworks and the ways they are usually carried out are built on a few dichotomies: academic versus societal spheres, quantitative versus qualitative approaches, and representative versus intervening analyses. We argue that these dichotomies contribute to a notion of academic achievement that is unrealistic, in a normative and descriptive sense. The concept of the "evaluative inquiry," as proposed here, amends the linear and individualised notion of academic work and its evaluation and discusses the implications of these moves for the work of the analyst. We suggest instead to understand academic achievement as distributed over a host of academic and non-academic participants to be studied by means of a portfolio approach. This approach to research evaluation requires a more engaged analyst who takes evaluation seriously as both an analytical and a strategic project.

\section{INTRODUCTION}

This paper introduces the evaluative inquiry, an approach that aims to challenge several dimensions of the current science system and the organisation of research evaluation, most notably its understandings of academic achievement, impact, and the ways these should be measured. It contributes to a "re-loading" of the term impact, drawing on the methodological and conceptual approaches of the social sciences and humanities in particular, and all the sciences in general (König et al., 2018). We propose a distributive understanding of academic achievement, thereby recognising the contributions of both academics and nonacademics. In addition, we put forth a portfolio approach to evaluation in order to detect the multiple realities that go into academic quality and in order to inspire conversations about these. Lastly, we move beyond approaches that claim to neutrally represent quality and relevance, by offering a style of strategic and collaborative intervention. We hope these three moves will help identify paths to reform and revitalise the science system and the normative, unilateral, and dichotomous ideals of excellence and impact.
The evaluative inquiry was proposed by Fochler and De Rijcke (2017) as a way to contribute to ongoing discussions about quality and relevance of research. Our team at CWTS (Leiden University, The Netherlands) has since then put the evaluative inquiry into practice in several projects, and this work informs this paper. Our ambition with these experiments in research evaluation is, in essence, to enable better conversations about academic value and its beneficiaries and rewards, rather than to further encourage "accounting for impact" (Rushforth and De Rijcke, 2015) by way of standardised formats and rankings.

\section{1) THE DUTCH CONTEXT}

To situate our approach, a few words are in order on the main characteristics of the Dutch higher education and science governance system. Like many other European countries, the Dutch higher education system distinguishes between two types of higher education institutions: universities on the one hand, and institutions for higher vocational education - so-called hogescholen - on the other. As a general rule, science governance instruments since the early 1980s have been built around a principle of "steering at a distance". The Netherlands in fact operate with what Richard Whitley (2007) has called a "weak" system of research evaluation, meaning that assessment results have no direct consequences for the distribution of funding to universities (in contrast to, for example, the UK). Rather, the principal strategy is to use formal evaluation as opportunities for self-reflection and organisational learning (see also Youtie and Corley, 2011; Hansson and Monsted, 2012). Conceptually, institutional research evaluation systems can serve three main purposes: a distributive, an improvement, and a controlling use (Molas-Gallart, 2012). In the Dutch context, the purpose of evaluation is clearly focused on improvement, and an evaluation can also spark organisational change. As Molas-Gallart puts it, "[a]n improvement use will focus on deriving lessons from the past experience to adapt the activities conducted to what evaluation studies will conclude is better practice. The improvement purpose is therefore relying on the existence of feedback mechanisms and the operational flexibility needed to function as a learning organization." (ibid, 589) We would suggest that an improvement-oriented evaluation system like the Dutch one provides particular opportunities for experimenting with evaluative inquiries. 
The Netherlands introduced a formal evaluation system as early as 1982. All research units at Dutch universities (as well as the institutes of the Royal Netherlands Academy of Arts and Sciences and the Netherlands Organisation for Scientific Research) are required to undergo an assessment in six-yearly intervals (see figure 1). The assessment consists of a peer review procedure by an external committee, involving personal site visits, interviews, and a review of research output and other activities. Halfway between the 6-yearly national research assessments, research units are moreover required to conduct a mid-term evaluation. The results of a self-evaluation serve as input for the assessment, and are also meant to encourage continuous self-monitoring of individual institutions. Another important change introduced in 2015 was that organisational responsibility for evaluation was decentralised and delegated to individual institutions, thus providing them with a greater degree of administrative discretion.

The exact modalities of assessment are outlined in the so-called "Standard Evaluation Protocol" (SEP). According to the SEP, institutions are required to provide a range of materials as input for the assessment, including inter alia a formal documentation of output and "performance indicators" (e.g., a complete list of publications, number of successfully defended $\mathrm{PhD}$ theses etc.), a description of the financing of a given research unit, and a qualitative narrative summarising the results and societal relevance of the research (see table 1 below). Whereas evaluation has traditionally placed an important emphasis on quantity of output and the perceived prestige of publications and research grants, the 2015 iteration of the SEP introduces a stronger emphasis on "societal relevance" of research, i.e. the engagement with non-academic audiences and partners. Academic excellence and societal relevance are however kept largely separate in the evaluative framework. Research units are ultimately graded according to a four-tiered scale (from "world-leading" to "unsatisfactory").

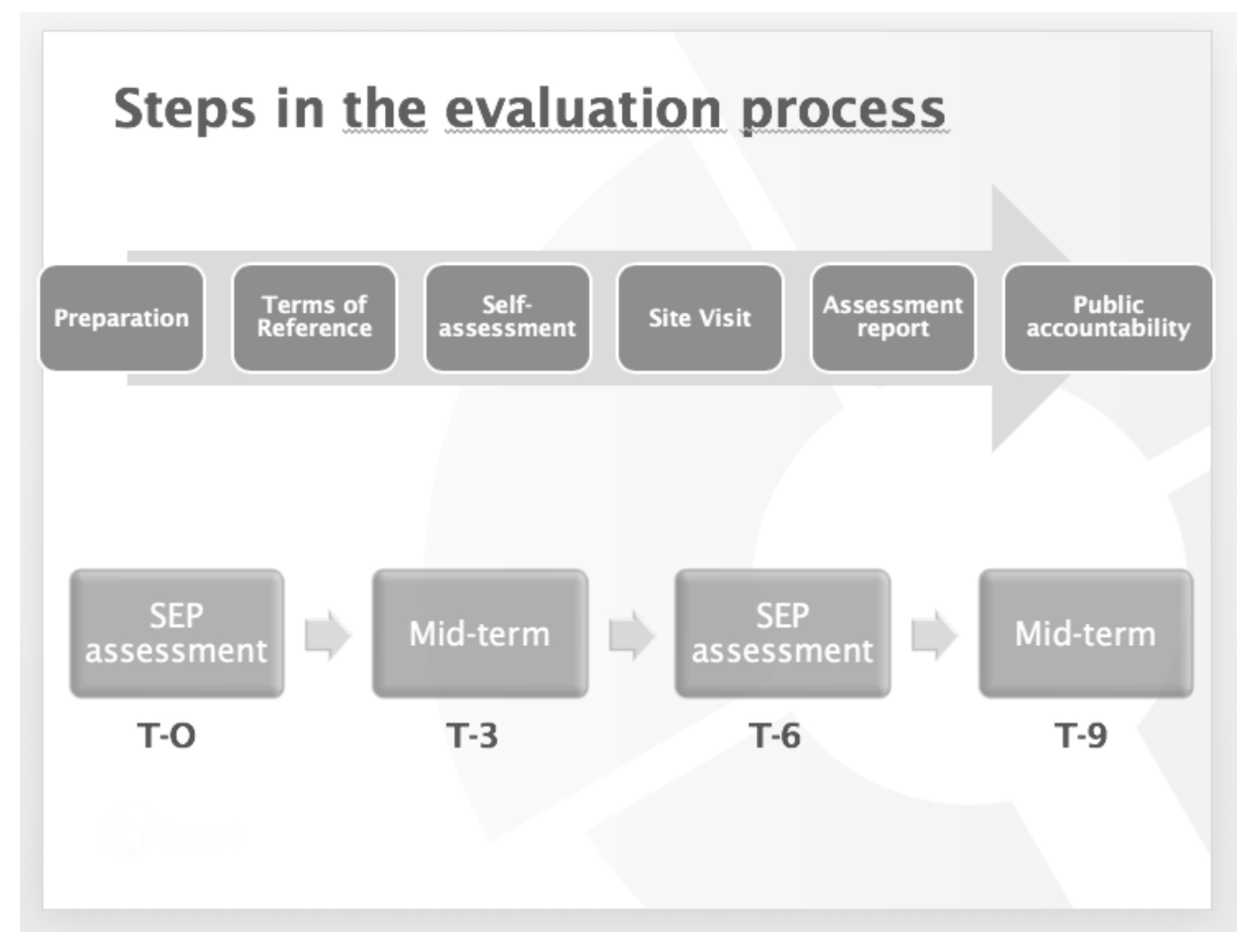

Figure 1. Steps in the Dutch evaluation process.

\begin{tabular}{|l|l|}
\hline $\begin{array}{l}\text { Description of unit's } \\
\text { organisational structure. }\end{array}$ & $\begin{array}{l}\text { Most important (and relevant) } \\
\text { performance indicators. }\end{array}$ \\
\hline Description of unit's financing. & $\begin{array}{l}\text { Results research and societal relevance } \\
\text { past } 6 \text { years (latter in a narrative). }\end{array}$ \\
\hline Strategy past 6 years. & $\begin{array}{l}\text { Link results to SEP criteria (quality, } \\
\text { relevance, viability). }\end{array}$ \\
\hline $\begin{array}{l}\text { Targets past } 6 \text { years (research, } \\
\text { societal relevance). }\end{array}$ & Strategy and targets next 5-10 years. \\
\hline
\end{tabular}

\begin{tabular}{|l|l|}
\hline $\begin{array}{l}\text { Relevant environmental factors } \\
\text { and developments past six years. }\end{array}$ & PhD Programme(s) \\
\hline $\begin{array}{l}\text { SWOT (Strengths, Weaknesses } \\
\text { Opportunities, Threats) analysis } \\
\text { and benchmarking. }\end{array}$ & Research integrity \\
\hline
\end{tabular}

Table 1: Formal requirements for self-assessment report (SEP 20152021). 


\section{2) EVALUATION AS A KNOWLEDGE MAKING PROJECT}

The evaluative inquiry was first introduced as a prompt to more enabling rather than reductive accounts of assessment by Fochler and de Rijcke (2017). The evaluative inquiry understands academic work as a process in which a variety of actors (including non-academic ones) are part of the sociotechnical networks through which knowledge is generated. In emphasising process and engagement rather than rating and ranking, it wants to bring to light the way quality is created and negotiated among multiple participants and amidst multiple epistemic commitments, rather than attributing it to individuals' actions and intentions who are subsequently compared. As such it is aligned with scholarly work that is interested in academic work and quality as it comes into being in interactions between values and networks of people, outputs, and resources (e.g., Bozeman and Sarewitz, 2011; de Jong et al., 2014; Matt et al., 2016; Prins and Spaapen, 2017).

Our team at CWTS is putting the evaluative inquiry into practice in several projects. On the basis of this work, we further specify the inquiry in relation to three contentious issues within the current science system and its evaluation: the much-debated dichotomy between academic and societal realms, the distinction between quantitative and qualitative approaches, and the allegedly invisible and neutral evaluating analyst.

The first issue we identify is the underlying divide in many evaluation frameworks between the academic and the societal. The inclusion of broader impacts into frameworks that originally put most emphasis on academic work has done justice to the interconnections between science and society that are especially strong for the social sciences and humanities. However, the way this relation is often imagined is problematic (cf. Calvert, 2006; de Jong et al., 2014; Felt and Wynne, 2007; Nowotny et al., 2001). It is often envisioned as a linear model of scientific knowledge production that starts with fundamental research and discovery, and ends with innovations that are beneficial for society through translational and applied research. Within this model, the influence of knowledge in society could be traced back to original inventions, the ultimate value of which can then be established. A problem with this model is that the individual (scholar or research institute) remains the locus of both value and responsibility now not only for academic publications, but also for producing societal relevance (Holtrop, forthcoming). Rather than realising that academic work frequently entails engagement with societal actors - and therefore one could argue that both relevance and quality originate in that interaction - one now has to write excellent papers and perform in societally relevant ways as well. The evaluative inquiry problematises the notion of a passive public audience that reaps the benefits of academic expertise, and instead highlights the "productive interactions" (Spaapen and van Drooge, 2011) between diverse stakeholders, and the distributed nature of academic achievement more generally. Regarding the assessment of impact, this would at least entail that audiences are seen not only as (co)producers of knowledge and its impact, but also as (co)producers of the criteria by which such impact is to be evaluated.

The unhelpful divide between spheres and stakeholders is perpetuated by another unhelpful divide: the one between quantitative and qualitative evaluation methods. We recognise the work done in academic and professional environments to problematise reliance on metrics and citation scores alone, and that argues that quantitative and qualitative methods are implicated in one another (cf Callon and Law, 2005). Moreover, initiatives such as the "Leiden Manifesto" (Hicks et al., 2015), the "Metric Tide" (Wilsdon et al., 2015) and "DORA", have presented careful responses and suggestions for next steps. We feel akin to these initiatives, and wish to stay away from the unproductive dichotomy of quantitative and qualitative methods. In our contribution, we move from a fixation on "getting it right" in evaluations, to an approach that presents research numerically, verbally, and/or visually in ways that make visible the complexity of actual practice and its engagements (Fochler and De Rijcke, 2017). This means that evaluative inquiry treats knowledge production as heterarchical (Stark, 2011): it sees phenomena as amenable to multiple orders of worth, rather than as connected to one rank order with clear winners and losers.

Our understanding of the enterprise of academic evaluation changes while we move from a linear model of academic achievement evidenced by individual actions and intentions to an understanding of academic value as situated within multiple epistemic commitments and relations between many actors. Evaluations are now no projects that look into academic worlds from the outside while taking stock of the valuables. Instead they are themselves knowledge producing endeavors, transforming evaluators and analysts into collaborators alongside evaluees. This is the third dichotomy that the evaluative inquiry wants to unsettle: the one between a detached analyst doing representations objectively on the outside and an engaged analyst located within. We build on a previous work that problematises the claim to detachment, objectivity, and neutrality that has characterised dominant modes of research evaluation (Candea et al., 2015; Daston and Galison, 2007). Instead, we take seriously that the act of representing quality is also an intervention (De Rijcke and Rushforth, 2015).

\section{3) THE INQUIRY}

Central to the evaluative inquiry is an understanding of academic achievement as distributed over a host of academic and non-academic participants. These achievements are to be studied by means of a coproduced portfolio approach, tailored to specific research units and evaluation purposes. Each method has its own strong points when it comes to detecting and amplifying reality (Law, 2004). Rather than advocating a combination of methods with the purpose of coming to more accurate representations of academic work, we argue that co-production and multiplication of methods allows for more interesting conversations about academic quality, and offers points of departure for strategically addressing all too real issues of power, money and reputation that are part of academic evaluation. Though the inquiry remains concerned with reaching an adequate understanding of academic achievement (or quality) in the analysis, the approach actively seeks to avoid reproducing the familiar role of the analyst as a detached accountant. Recognising evaluation itself as both an analytical and a strategic project, the analyst thus moves from objective observer into the role of an engaged evaluation expert, not only engaging in the analysis of quality but also in the analysis of the broader political projects of accountability with which it is intertwined. More than working towards a definitive report where research units are assessed on the basis of a predefined set of characteristics, the inquiry is set up to study, map and trace the research themes, pathways and productive interactions around the research unit through 
a portfolio of methods. Practically, the inquiry takes the form of three consecutive phases (see table 2).

\begin{tabular}{|l|l|}
\hline Phases & Approach \\
\hline 1. Exploration & $\begin{array}{l}\text { What is at stake in the assessment? } \\
\text { Questions addressed in document analyses } \\
\text { and interviews with management. }\end{array}$ \\
\cline { 2 - 3 } & $\begin{array}{l}\text { Design of research approach, choice } \\
\text { of combination of methods. }\end{array}$ \\
\hline 2. Data collection and Analysis & $\begin{array}{l}\text { \# (e.g.) "Contextual Response Analysis" (Prins, n.d.); } \\
\text { contextual scientometrics (Waltman and van Eck, } \\
\text { 2016); bibliographic coupling; co-citation analyses; } \\
\text { "Area Based Connectedness" (Noyons, 2018) } \\
\text { \# Interviews with researchers and stakeholders } \\
\text { regarding organisational and academic themes, } \\
\text { operationalisation, outputs and impacts. }\end{array}$ \\
\cline { 2 - 3 } & $\begin{array}{l}\text { Workshops - to test hypotheses, present themes } \\
\text { and pathways, collect more input for SWOT-analysis. }\end{array}$ \\
\hline 3. Reporting & $\begin{array}{l}\text { Analysis in terms of organisational issues } \\
\text { and academic ambitions and themes. SWOT. } \\
\text { Suggestions as to how to write the self-evaluation. }\end{array}$ \\
\hline
\end{tabular}

Table 2: Phases in an evaluative inquiry.

An evaluative inquiry is geared towards detecting lively interactions and outcomes, which can be enveloped in themes and 'pathways' (loosely based on Matt et al., 2016), thereby connecting academic and societal domains. In past projects, we have built these themes and pathways by using a combination of quantitative methods, interviews and workshops to collect information about the themes people work on, the resources and people that are mobilised in their research projects, the highly varied outputs that are generated as a result, and the way these outcomes travel elsewhere into other academic, professional or societal realms (see table 2). Though the inquiry allows for different combinations of methods, one form this combination can take is that of a generative dialogue. Interviews and quantitative analyses are first used to gather information, for example with regards to themes that researchers and stakeholders consider central to the work and the variable audiences that it reaches (or fails to reach). The outcomes of this first round of inquiry are used to identify a first set of possible themes and pathways, which are then presented back to the research unit, for example in a collective workshop. The presentation is meant to elicit further response, and allows those involved to think with and elaborate on the first results in a collective setting with colleagues, stakeholders and the analysts. The final report is subsequently written in terms of the organisational issues and the academic ambitions and themes that have emerged. This could for example include an interactive analysis and visualisation of prevalent themes and ambitions, their operationalisation, the people and resources that are mobilised, the outputs this generates and the way these are cited, used, and travel further into the world. The outcomes of this type of detailed interaction with individual researchers, research leaders and their work can be added to the information that is gathered in the analysis of organisational issues represented in a SWOT. Organisational documents and data can be combined with insights gained in individual interviews or workshops, adding additional depth and possibly room for creative synergy between people and data. Crucially, these processes and roles are scripted together, so as to enable both a highly rigorous and a highly grounded analysis.
The subsequent self-assessment document is authored by the research unit itself. Our report is written in such a way that it can be a conversation piece and offers openings for discussion - internally, and with other academic institutes, science policy environments and stakeholders interested in academic quality. The inquiry can, but not necessarily does, fix the state of the object of evaluation in a definitive account. Moreover, the outcomes of the inquiry are in this sense not limited to the report. Individual elements of the evaluative inquiry itself, like the workshop, are excellent tools to bring the organisation and/or stakeholders together and collaboratively identify problems, make tough decisions, work on solutions, or plan for the future. They trigger meaningful conversations about how to deal with pressing challenges such as the increasing roles and demands of peer communities, professional and societal partners, government or industry while building on individual and institutional strengths. The plurality of actors involved can take on a variety of roles throughout the inquiry. Staff members and stakeholders can be consciously drawn into the production process, being in some ways the experts and authoritative analysts on the values and interrelations of the work. The analysts, in turn, are more than outsiders who merely "run" pre-set quantitative or qualitative analyses: they become active co-producers of the inquiry.

\section{DISCUSSION: EVALUATION AS KNOWLEDGE PRODUCTION}

The notion of "impact" suggests an interaction of which the source, target and content are clearly identifiable. Traditional frameworks for evaluation tend to focus on whether and to what extent such impact is achieved. The concept of evaluative inquiry, as developed in this paper, revises this linear notion of impact as the central precept of research evaluation. The framework builds on work by e.g. Matt et al. (2016), Spaapen and Van Drooge (2011), and Prins and Spaapen (2017), by conceptualising scholarly work not in terms of a linear diffusion of knowledge, but rather as an emergent effect of an unfolding, multidirectional research process. Evaluative inquiry reveals the epistemic commitments and community values of local practices. It thus essentially approaches evaluation as a knowledge production process. From this starting point, our approach to evaluation sees the relevance of scientific work as an unfolding process, in which a variety of academic and non-academic actors are involved. This approach emphasises process and engagement rather than accounting and ranking. Crucially, evaluative inquiry identifies values, networks of people, and resources as collectives. It helps articulate how "worlds" are created and negotiated in relation to these values.

With evaluative inquiry, we thus move away from evaluations as detached, clear delineations of academic value. Researchers obviously do not just produce excellent research or articles in journals, or even knowledge that is of use for society at large. More than that, they are prominent world-makers, and their knowledge has consequences for the world they and others inhabit as well as their experiences in it. This suggests that diverse (relational, communicative, organisational) values, activities and outcomes have to be taken into account in evaluations. Conventional approaches to research assessment treat these values hierarchically. This works well within accountability systems that embed actors and actions in fixed and calculable value regimes. Both bibliometrically framed assessments and assessments of societal impact operate through a quite 
similar logic of "return-on-investment". An evaluative inquiry, in contrast, is not meant to result in one definitive document that "makes up the account". In the mode of evaluative inquiry, standardisation is less relevant than staying close to the epistemic missions, frictions and resonances of academic work. The approach understands academic performance or impact as an effect of translations within and between networks of actors that make up academic research and its environments (cf. Matt et al., 2016). The aim is to find out what are the central issues or ambitions, how they are operationalised, what kind of outcomes this yields and where the outcomes travel to. Evaluative inquiries broaden our understanding of what counts as academic achievement. They afford a greater inclusivity of research settings, which ideally means a more meaningful treatment of the social sciences and humanities. As such, our contribution is meant to give space to, and reinforce the greater role to be played by, the social sciences and humanities, including the fields of research evaluation and scientometrics. The social sciences and humanities have the conceptual tools to enrich the methodological portfolio for gathering information about the worlds that academics inhabit and contribute to. A pluralisation of perspectives and methods enriches the inquiry by opening up what can be talked about in evaluations. This pluralisation should not happen in secluded spaces such as scientometric labs, but with the participation of stakeholders, so as to take in consideration their contexts (cf. Rafols, 2018). Furthermore, evaluative inquiry is sensitive to how value systems might differ across teams an organisations, and evolve over time. This approach makes it possible to articulate positions, roles and values that are subordinate to dominant currents in academic practice and that are often silenced in traditional evaluations.

In conclusion, with the evaluative inquiry we fully subscribe to the call for "re-loading" the notion of impact (König et al., 2018), a notion with problematic ballistic connotations. We hope our contribution feeds ongoing discussions among academics, policy-makers, and other stakeholders about the fault lines between forms of value, the uncertainties in evaluating, and the politics of formats, protocols and endings. Our contribution is a strong plea to create more room for experiments in research evaluation (and it is clear that we are not done experimenting ourselves). We think this simultaneously entails: 1) advocating and conducting rigorous analytical work; 2) a willingness of those under assessment to be open to more engaged modes of assessment; 3 ) using the full potential of the form(s) evaluation can take; and 4) using quantitative methods in much more interesting ways. Rather than taking an a-priori, reductive approach to what counts in research evaluation - think of the proliferation of publication lists and performance metrics - it is much more useful to produce and present the multiple meanings and purposes of research. Evaluative inquiry takes evaluation itself as a deliberative, generative process of knowledge production in its own right. In doing so, it opens up more than one way for empirical data, evaluators, and other actors to be implicated in the evaluation. The generative capacity of the inquiry is partially built on keeping more open the roles of the various coproducers, and the evaluative criteria that may be generated from their variable positions. This also means that the legitimacy of the evaluation is not solely based on the analyst's correct implementation of criteria, but much more so on the degree to which co-producers think that the process and results do justice to their joint work.

\section{REFERENCES}

Association of Universities in the Netherlands (VSNU), the Netherlands Organisation for Scientific Research (NWO) and the Royal Netherlands Academy of Arts and Sciences (KNAW). Standard Evaluation Protocol (SEP) 2015-2021. Protocol for Research Assessments in the Netherlands.

Bozeman, B. and Sarewitz, D. (2011). Public Value Mapping and Science Policy Evaluation. Minerva, 49(1), 1-23.

Calvert, J. (2006). What's Special about Basic Research? Science, Technology \& Human Values, 31(2), 199-220.

Callon, M. and Law, J. (2005). On Qualculation, Agency and Otherness. Environment and Planning D: Society and Space, 23(5), 717-33.

Candea M., J. Cook, C. Trundle and Yarrow T. (Ed.) (2015). Detachment: Essays on the Limits of Relational Thinking. Manchester: Manchester University Press.

Daston, L. and Galison, P. (2007). Objectivity. Cambridge, The MIT Press.

De Jong, S., Barker, K., Cox, D., Sveinsdottir, T. and van den Besselaar, P. (2014). Understanding societal impact through productive interactions: ICT research as a case. Research Evaluation, 23(2): 89-102.

De Rijcke, S. and Rushforth, A. (2015). To intervene or not to intervene, is that the question? On the role of scientometrics in research evaluation. JASIST, 66 (9), 1954-1958.

Felt, U. and Wynne, B. (2007). Taking European Knowledge Society Seriously. Report of the Expert Group on Science and Governance to the Science, Economy and Society Directorate, Directorate-General for Research, European Commission. Luxembourg: Office for Official Publications of the European Communities. Retrieved November 1st, 2018 from: https://ec.europa.eu/research/science-society/document_library/ pdf_06/european-knowledge-society_en.pdf

Fochler, M. and De Rijcke, S. (2017). Implicated in the Indicator Game? An Experimental Debate. Engaging Science, Technology, and Society, 3, 21-40.

Hansson, F. and Monsted, M. (2012). Changing the peer review or changing the peers - Recent developments in assessment of large research collaborations. Higher Education Policy, 25(3): 361-379.

Holtrop, T.J. (forthcoming). The Evaluative Inquiry: A New Approach to Research Evaluation. Blogpost, forthcoming at http://www.cwts.nl/blog.

König, T., Nowotny, H. and Schuch, K. (2018). Impact Re-loaded. Pathways to impact from SSH research. CfP SSH Impact Conference Vienna. Retrieved 2 November, 2018 from: https://www.ssh-impact.eu/ wp-content/uploads/2018/06/Impact_Re-loaded_180614.pdf

Law, J. (2004). After Method: Mess in Social Science Research. London: Routledge.

Matt, M., Gaunand, A., Joly, P.-B. and Colinet. L. (2016). Opening the black box of impact - Ideal-type impact pathways in a public agricultural 
research organization. Research Policy, 46, 207-218.

Molas-Gallart, J. (2012). Research Governance and the Role of Evaluation: A Comparative Study. American Journal of Evaluation, 33(4), 583-98.

Molas-Gallart, J., D'Este, P., Llopis, 0. and Rafols, I. (2015). 'Towards an alternative framework for the evaluation of translational research initiatives', Research Evaluation, 25(3), 235-243.

Nowotny, H., Scott, P. and Gibbons, M. (2001). Rethinking science: knowledge in an age of uncertainty. Cambridge: Polity.

Noyons, E. (2018). Monitoring how science finds its way into society: measuring societal impact through area-based connectedness (ABC). Blogpost. Retrieved November 1st, 2018 from: https://www.cwts.nl/ blog?article $=n-r 2 u 264 \&$ title $=$ monitoring-how-science-finds-its-wayinto-society-measuring-societal-impact-through-area-based-connectedness-abc

Prins, A. A. M. and Spaapen, J. (2017). Serving variegated audiences: From ranking oriented evaluation to mission oriented evaluation. fteval Journal for Research and Technology Policy Evaluation, 44 (September), $42-49$.

Prins, A. (n.d.). Contextual Response Analysis. Retrieved November 1st, 2018 from http://www.adprins.nl/index.php?id=box2

Rabinow, P. (2011). The Accompaniment: assembling the contemporary. Chicago: University of Chicago Press.

Rafols, I. (2018). S\&T indicators 'in the wild': contextualisation and participation for responsible metrics. Blogpost. Retrieved November 3rd, 2018 from: https://www.cwts.nl/blog?article=n-r2u2548title=st-indicatorsin-the-wild-contextualisation-and-participation-for-responsible-metrics

Rushforth, A.D. and De Rijcke, S. (2015). Accounting for Impact? The Journal Impact Factor and the Making of Biomedical Research in the Netherlands. Minerva, 53, 117-139.

Spaapen, J. and Van Drooge, L. (2011). Introducing Productive Interactions in Social Impact Assessment. Research Evaluation, 20 (3), 211-18.

Stark, D. (2011). The Sense of Dissonance: Accounts of Worth in Economic Life. Princeton, NJ: Princeton University Press

Waltman, L., and Van Eck, N. J. (2016). The Need for Contextualized Scientometric Analysis: An Opinion Paper. In I. Ràfols, J. Molas-Gallart, E. Castro-Martínez, and R. Woolley (Eds.), Proceedings of the STI Conference 2016. Peripheries, Frontiers and beyond (pp. 1-9). Valencia, Spain: Universitat Politècnica de València.

Youtie, J. and Corley, E. (2011) Federally Sponsored Multidisciplinary Research Centers: Learning, Evaluation, and Vicious Circles. Evaluation and Program Planning, 34, 13-20.

Whitley, R. (2007). Changing Governance of the Public Sciences. In R. Whitley and J. Glaeser (Eds), The Changing Governance of the Sciences.
Sociology of the Sciences Yearbook (pp. 3-27). Dordrecht: Springer.

\section{AUTHORS}

[Numbers] CASRAI CRediT taxonomy for contributor roles (https://casrai. org/credit/)

\section{SARAH DE RIJCKE $[1,6,7,10,12,13,14]$}

Centre for Science and Technology Studies (CWTS), Leiden University P.0.Box 905, Leiden, 2300 AX (The Netherlands)

E: s.de.rijcke@cwts.leidenuniv.nl

\section{TJITSKE HOLTROP $[1,5,6,13,14]$}

Centre for Science and Technology Studies (CWTS), Leiden University P.0.Box 905, Leiden, 2300 AX (The Netherlands)

E: t.j.holtrop@cwts.leidenuniv.nl

\section{WOLFGANG KALTENBRUNNER ${ }^{[13,14]}$}

Centre for Science and Technology Studies (CWTS), Leiden University P.0.Box 905, Leiden, 2300 AX (The Netherlands)

E:w.kaltenbrunner@cwts.leidenuniv.nl

\section{JOCHEM ZUIJDERWIJK ${ }^{[5,6,13,14]}$}

Centre for Science and Technology Studies (CWTS), Leiden University P.0.Box 905, Leiden, 2300 AX (The Netherlands)

E: j.b.zuijderwijk@cwts.leidenuniv.nl

\section{ANNE BEAULIEU ${ }^{[13]}$}

University of Groningen, Campus Fryslan (CF)

Sophialaan 1, 8911 AE, Leeuwarden (The Netherlands) and

CWTS, Leiden University

P.0.Box 905, Leiden, 2300 AX (The Netherlands)

E: j.a.beaulieu@rug.nl

E: j.a.beaulieu@cwts.leidenuniv.nl

\section{THOMAS FRANSSEN $[5,6,13]$}

Centre for Science and Technology Studies (CWTS), Leiden University P.0.Box 905, Leiden, 2300 AX (The Netherlands)

E: t.p.franssen@cwts.leidenuniv.nl

\section{THED VAN LEEUWEN ${ }^{*}[5,6,13]$}

Centre for Science and Technology Studies (CWTS), Leiden University P.0.Box 905, Leiden, 2300 AX (The Netherlands)

E: leeuwen@cwts.leidenuniv.nl

\section{PHILIPPE MONGEON ${ }^{[13]}$}

Danish Centre for Studies in Research and Research Policy, Aarhus

University

Bartholins Allé 7

DK-8000, Aarhus, C, Denmark and

CWTS, Leiden University

P.0.Box 905, Leiden, 2300 AX (The Netherlands)

E:philippe.mongeon@ps.au.dk

E: p.mongeon@cwts.leidenuniv.nl

\section{CLIFFORD TATUM[1, 13]}

Centre for Science and Technology Studies (CWTS), Leiden University 
P.0.Box 905, Leiden, 2300 AX (The Netherlands)

E: c.c.tatum@cwts.leidenuniv.nl

\section{GOVERT VALKENBURG ${ }^{[13]}$}

Department of Interdisciplinary Studies of Culture, Norwegian University of Science and Technology (NTNU)

N0-7491 Trondheim, Norway

E: govert.valkenburg@ntnu.no

\section{PAUL WOUTERS ${ }^{[13]}$}

Centre for Science and Technology Studies (CWTS), Leiden University P.0.Box 905, Leiden, 2300 AX (The Netherlands)

E: p.f.wouters@cwts.leidenuniv.nl

\section{KEYWORDS:}

Research evaluation; societal relevance; intervention; mixed-methods; engagement; heterogeneity 